\title{
A dor como um problema psicofísico*
}

Pain as a psychophysical problem

José Aparecido da Silva ${ }^{1}$, Nilton Pinto Ribeiro-Filho²

${ }^{*}$ Recebido do Laboratório de Psicofísica e Percepção. Ribeirão Preto, SP.

\section{RESUMO}

JUSTIFICATIVA E OBJETIVOS: A quantificação da dor enfrenta dificuldades especiais. Elas surgem devido à óbvia associação das sensações de dor com um conjunto de fatores emocionais, motivacionais e culturais. Apesar disso, a mensuração da dor é essencial para a avaliação e o tratamento dos seus efeitos. O objetivo deste estudo foi mostrar que os métodos psicofísicos podem ser adequadamente empregados para a mensuração, tanto da dor clínica quando da dor experimental.

CONTEÚDO: Em adição, a metodologia pode ser útil para analisar os mecanismos da dor, a analgesia, os vieses metodológicos inerentes aos registros verbais da dor e dissociar os componentes sensoriais e cognitivos da sensação/ percepção de dor.

CONCLUSÃO: A metodologia psicofísica pode ser uma medida fidedigna e válida do quinto sinal vital, que é a dor, em todas as suas dimensões.

Descritores: Avaliação da dor, Dor clínica, Dor experimental, Mensuração da dor, Percepção da dor, Psicofísica da dor.

\section{SUMMARY}

BACKGROUND AND OBJECTIVES: Pain quantification faces special difficulties. They appear due to the

1. Professor Titular do Departamento de Psicologia da Universidade de São Paulo, Campus da USP. Ribeirão Preto, SP, Brasil.

2. Professor Adjunto do Instituto de Psicologia da Universidade Federal do Rio de Janeiro, Praia Vermelha. Rio de Janeiro, RJ, Brasil.

Endereço para correspondência:

José Aparecido da Silva

Avenida dos Bandeirantes, 3900

14040-901 Ribeirão Preto, SP.

Fones: (16) 3602-3728 - (16) 9991-8443

E-mail: jadsilva@ffclrp.usp.br obvious association of pain to a set of emotional, motivational and cultural factors. However, pain measurement is essential to evaluate and treat its effects. This study aimed at showing that psychophysical methods might be adequately used to measure both clinical and experimental pain.

CONTENTS: In addition, the methodology may be useful to analyze pain mechanisms, analgesia, methodological biases inherent to pain verbal records, and to dissociate sensory and cognitive components from pain sensation/ perception.

CONCLUSION: The psychophysical methodology may be a trustworthy and valid measurement of the fifth vital sign, which is pain, in all its dimensions.

Keywords: Clinical pain, Experimental pain, Pain evaluation, Pain measurement, Pain perception, Pain psychophysics.

\section{INTRODUÇÃO}

A sensação de dor é fundamental para a sobrevivência. Dor é o primeiro indicador de qualquer lesão tecidual. Qualquer estímulo que resulta em lesão ou ferimento conduz a uma sensação de dor, entre eles o calor, o frio, a pressão, a corrente elétrica, os irritantes químicos e até mesmo os movimentos bruscos. Diferente de outros sistemas sensoriais, todavia, o sistema sensorial para a dor é extremamente amplo; uma sensação dolorosa pode ser iniciada em qualquer parte do corpo ou no próprio sistema nervoso central (SNC). Vários locais são emparelhados aos vários tipos de sensações de dor. A sua percepção é claramente uma rica e multidimensional experiência, a qual varia tanto em qualidade quanto em intensidade sensorial, assim como em suas características afetivo-motivacionais.

Sem dúvida, a sensação de dor é um importante domínio da experiência humana e, talvez, aquela que tenha, continuamente e com mais sucesso, iludido inúmeras tentativas de conceituação consistente, de quantificação, ou mesmo de documentação sistemática, por ge- 
rações de especialistas de diferentes áreas do conhecimento. Infelizmente, ela ainda possui o mesmo status, apesar do renovado interesse no entendimento de sua natureza, agora agregado com a aplicação de uma quantidade enorme de diferentes e inovados métodos de experimentação e análises. Sua complexidade e natureza multidimensional, as quais são evidentes mesmo nas análises mais elementares dos vários tipos de dor, têm, contudo, obstruído virtualmente o desenvolvimento de uma definição adequada de dor, ou o que, talvez, seja o mais importante, dificultado a construção de uma teoria geral da dor, bem como a derivação de técnicas de tratamento claramente eficazes.

Uma parte do diálogo mantido entre um médico de um time de futebol, e um grupo de jornalistas, que cobria o dia a dia dos jogadores, revela como o estudo da dor é complexo e intrigante. “... Eu sempre aceitei a dor desse jogador, mesmo achando que os exames clínicos eram normais. Mas a dor é um artefato subjetivo...", comentou o médico.

Não obstante essa complexidade e dificuldades para entender a natureza do fenômeno da dor, sua análise deve, necessariamente, ser concebida dentro do contexto fundamental de uma relação, ainda que incompleta, entre estímulo e sensação. O problema pode ser colocado de maneira mais simples, ater-se em apenas duas alternativas para o aparecimento do estímulo adequado (presente ou ausente) e, também, em duas alternativas para o registro da experiência da dor (presente ou ausente). Assim, o problema da avaliação e da mensuração da dor torna-se genuinamente um problema psicofísico, envolvendo a detecção, a discriminação e a magnitude da sensação a estímulos dolorosos.

A mensuração da dor é provavelmente uma das áreas mais importantes no vasto domínio do estudo da dor. Os pioneiros na pesquisa acerca da dor, interessados em sua mensuração, adaptaram a metodologia psicofísica com o propósito de avaliar e mensurar a dor clínica e a dor experimental. De fato, os métodos atuais utilizados para a mensuração e avaliação da dor têm raízes históricas na psicofísica, um campo da psicologia experimental que de longa data se preocupa com as relações entre as propriedades dos estímulos e as respostas ou reações comportamentais, ou percepções sensoriais. A psicofísica tem como suposição central que o sistema perceptual é um instrumento de mensuração, a gerar resultados (experiências, julgamentos, respostas) que podem ser sistematicamente mensurados e analisados. Os estímulos podem ser métricos (têm unidades físicas definidas) e não métricos (sem unidades físicas). Por isso a psicofísica, analisando os componentes sen- soriais, hedônicos e cognitivos da dor, tem sido muito importante para o melhoramento da avaliação da dor, principalmente no sentido de fornecer métodos para a mensuração diferencial dessas variadas dimensões psicológicas da experiência e percepção da dor.

De fato, as aplicações da psicofísica têm sido relevantes tanto para o tratamento, quanto para a manipulação da dor aguda e da dor crônica. A psicofísica da dor tem tido um papel destacado em clarificar os mecanismos da dor e, também, em fornecer uma base científica para os modernos métodos de avaliação e mensuração da dor. Como as medidas da dor são essenciais para a avaliação, bem como para o tratamento dos efeitos da dor, o rigor das ferramentas da psicofísica pode contribuir para este processo de mensuração e avaliação tanto da dor clínica quanto da dor experimental. Elas também podem ser úteis para analisar os mecanismos da dor, a analgesia, assim como para investigar os inúmeros vieses inerentes aos registros verbais da dor. Além disso, a metodologia psicofísica pode contribuir para a análise e acompanhamento das técnicas e estratégias de tratamentos usadas para alívio da dor ${ }^{1}$. Há, porém, inúmeros problemas metodológicos referentes aos diferentes métodos ou procedimentos utilizados para a sua adequada avaliação. Apesar disso, a metodologia psicofísica pode gerar uma medida fidedigna e válida do quinto sinal vital - a dor - em suas três principais dimensões, ou seja, sensorial, avaliativa e afetiva.

\section{DEFINIÇÃO DA DOR}

Como já descrito, a dor pode ser definida como uma experiência subjetiva que pode estar associada à lesão real ou potencial nos tecidos, podendo ser descrita tanto em termos destas lesões quanto por ambas as características. Independente da aceitação e da amplitude dessa definição, a dor é considerada como uma experiência, uma sensação, genuinamente subjetiva e pessoal. A dor tem aspectos sensoriais, afetivos, autonômicos e comportamentais. Além disso, a sensação de dor não necessariamente necessita ser baseada em qualquer experiência prévia com ela. A despeito disso muitos estudiosos tratam a dor como uma simples dimensão variando apenas na magnitude sensorial. Mas, descrevê-la somente em termos de sua intensidade é o mesmo que especificar o mundo visual apenas em termos da intensidade luminosa, sem considerar o padrão, a cor, a textura. Apenas uma definição que integra todas estas características da sensação de dor pode ser significativa no contexto clínico e de pesquisa. 


\section{DOR CLÍNICA VERSUS DOR EXPERIMENTAL}

Comparação sistemática entre a dor produzida no laboratório a dor experimental e a dor clínica tem atraído vários pesquisadores, tanto por razões teóricas quanto práticas. A dor experimental e a dor clínica diferem pelo menos na fonte do desconforto, e pelas reações afetivas e cognitivas do indivíduo. De fato, a dor experimental não implica qualquer envolvimento psicológico significativo para o sujeito e não há um sofrimento real, tal como ocorre com a dor clínica. Além disso, a duração da dor experimental - segundos, minutos, talvez um período tão longo quanto duas horas - é, invariavelmente, muito menor do que a dor clínica, a qual pode durar por muitos anos. A intensidade da dor experimental é usualmente muito menor do que a severidade da dor clínica.

Embora seja tecnicamente possível manipular a dor experimental para que ela produza padrões de sofrimento, angústia, ansiedade, duração e nível de intensidade os mais similares àqueles que ocorrem com a dor clínica, tais manipulações, felizmente, não são éticas e nem mesmo legais. Não obstante, a indução da dor possui algumas vantagens, pois permite um maior controle dos estímulos e das condições experimentais, combinados com um registro cuidadoso das respostas dos sujeitos. Isto certamente não ocorre com a dor clínica. Embora essas diferenças sejam extremamente valiosas, elas fracassam, por não destacarem a mais importante. Do ponto de vista psicofísico, isto se refere à natureza dos julgamentos que tipicamente aparecem nos dois contextos ou ambientes. No ambiente clínico, além do questionamento necessário para a documentação médica lidando especificamente com os aspectos qualitativos da experiência da dor, um paciente típico raramente, faz se em algum momento o faz, um julgamento explícito sobre a intensidade de sua sensação dolorosa. Em contraste, os estudos de laboratório da dor, quase que por definição, requerem que o sujeito concentre-se sobre seus sentimentos de dor e julgue a intensidade dos mesmos. Explicitamente, e de certa forma exclusivamente, o julgamento dos aspectos intensivos de uma dada experiência de dor claramente distingue a dor clínica da dor induzida em laboratório. Em adição, no contexto de laboratório é possível requerer do sujeito que ele faça julgamentos de várias intensidades de dor, apresentadas, aleatoriamente, em situações controladas de estimulação, enquanto no ambiente clínico o sujeito julga, se isso eventualmente ocorrer, uma dada dor que ele está vivenciando naquele momento vivenciando.
Mesmo naquelas observações clínicas onde aspectos intensivos da dor são de interesse, algumas vezes, certas medidas são obtidas indiretamente como, por exemplo, o número de pacientes que solicita medicação, a quantidade de narcóticos ou de outros analgésicos requeridos, o número de queixas, de remoções, etc., no lugar de ocorrerem avaliações explícitas. Esta diferença que, pode ser óbvia e fácil de ser negligenciada (e de fato ela é) do ponto de vista tradicional, é substancial do ponto de vista de uma abordagem psicofísica que considera tanto a avaliação (aspectos qualitativos variados) quanto à mensuração (aspectos quantitativos) da dor, tanto em ambientes clínicos como em ambientes de pesquisa. Talvez, tenha sido por esta razão que, atualmente, em alguns países, a dor foi declarada como o quinto sinal vital, a qual deve sempre ser mensurada, registrada, ou anotada de alguma forma.

Apesar das diferenças entre dor clínica e dor experimental, certamente há relações diretas e indiretas entre elas, as quais justificam a importância dos estudos de dor em laboratório. A metodologia psicofísica moderna criada por Stevens, quase que exclusivamente no contexto de laboratório com outras modalidades sensoriais, muito contribuiu para o desenvolvimento dos métodos escalares diretos que, atualmente, são freqüentemente utilizados na mensuração das diferentes dimensões da dor clínica.

A psicofísica clássica, com sua ênfase nos estímulos, foi muito útil para o desenvolvimento da mensuração da dor experimental, enquanto, ao contrário, a psicofísica moderna, com sua ênfase na sensação, muito contribuiu para a mensuração da dor clínica. Na dor clínica, o estímulo exato não é conhecido, ou se conhecido, não é facilmente manipulado, de maneira que o experimentador não tem controle direto sobre ele. Devido a essa interação, os resultados experimentais podem, eventualmente, contribuir para a avaliação objetiva e a predição das reações de dor, enriquecendo-as, especialmente no entendimento da dor crônica. Sem dúvida, inúmeros estudos têm revelado que os mesmos fatores que modelam as respostas aos estímulos que provocam dor experimental, também contribuem para a experiência da dor clínica, por exemplo, mais sensibilidade à dor é associada com maior intensidade de dor clínica ${ }^{1-3}$.

\section{POR QUE MENSURAR A DOR?}

Por ser uma experiência subjetiva, a sensação de dor não pode ser objetivamente determinada por instrumentos físicos que, usualmente, mensuram diretamen- 
te o peso corporal, a temperatura, a altura, a pressão arterial e a pulsação. Em outras palavras, não existe um instrumento padrão único e invariável, tal como uma régua, que permita a um observador externo mensurar de forma objetiva essa experiência interna, complexa e genuinamente pessoal. A despeito destas dificuldades intrínsecas, por que é importante mensurar a dor?

A mensuração da dor é extremamente importante no ambiente clínico, pois se torna impossível manipular um problema desta natureza sem ter uma medida sobre a qual basear o tratamento ou a conduta terapêutica. Sem tal medida, torna-se difícil determinar se um tratamento é necessário, se o tratamento prescrito é eficaz, ou mesmo quando deve ser interrompido um dado tratamento. Com uma mensuração apropriada da dor torna-se possível determinar se os riscos de um dado tratamento superam os danos causados pelo problema clínico e, também, permite escolher qual é o melhor e o mais seguro entre diferentes tipos de conduta terapêutica. Ao lado disso, pode-se fazer um melhor acompanhamento e análise dos mecanismos de ação de diferentes drogas farmacológicas. Em outras palavras, é possível aperfeiçoar a escolha. Considerar apenas a característica sensorial da dor, especialmente a sua intensidade, ignorando suas propriedades afetivas e motivacionais é olhar para apenas parte do problema, e, talvez, nem mesmo para a parte mais importante dele. Há tempos destacou que a experiência subjetiva de dor é mais importante do que qualquer outro aspecto associado a ela ${ }^{4}$.

Uma avaliação eficaz da intensidade da dor possibilita examinar sua natureza, suas origens e os seus correlatos clínicos em função das características emocionais, motivacionais, cognitivas, e de personalidade do cliente/paciente. Algumas vezes, apenas medidas grosseiras, tais como "dor presente" ou "dor ausente", são necessárias para as intervenções clínicas; mas, para completamente entender o fenômeno e para avaliar a eficácia destas intervenções, necessitamos de medidas mais sofisticadas tanto da intensidade quanto das respostas afetivas associadas à dor.

\section{A LINGUAGEM DA DOR}

Como já descrito, a dor é uma experiência descrita em termos de características sensoriais, motivacionais e cognitivas e, muitas vezes, com sequelas emocionais. Por isso a utilidade de muitas medidas da dor, como, por exemplo, as escalas e os questionários multidimensionais, resultam parcialmente do reconhecimento e da avaliação independente desses diferentes componentes ou dimensões da dor. Todavia, no passado, um grande número de estudos acerca da dor e da analgesia, e ainda atualmente realizados, consideram a dor como uma dimensão unitária que varia apenas na magnitude de sua intensidade. Mas, como os resultados de um experimento sobre a dor podem depender de qual dimensão de dor está sendo no momento da avaliação, o fato de usar de uma simples medida da sensação dolorosa pode introduzir significativa variabilidade sobre os mecanismos e também sobre o tratamento eficiente da dor.

$\mathrm{O}$ que os pacientes entendem quando eles descrevem a magnitude de dor que estão sentindo? Eles referem-se à intensidade sensorial, à presença de qualidades sensoriais específicas, ou ao seu sofrimento, ansiedade, angústia? São os registros de dor usualmente associados a uma dessas dimensões, ou os seus significados variam entre indivíduos? Se o estudo da sensação da dor precisa ter uma fundamentação científica, é essencial mensurá-la. Por exemplo, caso necessite conhecer a eficácia de diferentes fármacos analgésicos, precisa-se de números para dizer que a dor diminuiu de alguma forma. Além da importância de conhecer que um fármaco diminuiu a sua intensidade, também é importante conhecer se o fármaco especialmente diminuiu a qualidade de queimação da dor, ou se os sentimentos de desconforto e de desprazer, associados com a dor, sumiram de alguma forma ${ }^{5}$.

Ora, a avaliação da dor clínica é, usualmente, baseada nos registros verbais ou nos descritores comumente usados pelos pacientes para descreverem a dor que estão vivenciando naquele momento. Um problema que existe relaciona-se ao grau em que esses descritores verbais, comumente utilizados, compartilham os mesmos significados entre as principais dimensões da dor. Isto porque um dado descritor de dor pode ter mais do que um significado associado a ele. Isto levou, por exemplo ${ }^{6}$, a desenvolverem o questionário de avaliação de dor McGill como um instrumento para avaliar as qualidades sensoriais, afetivas e avaliativas da dor, juntamente com vários outros aspectos, tais como intensidade, padrão e localização. Este questionário tem sido traduzido e padronizado para diferentes culturas, raças e sexos, haja vista a grande variabilidade no significado dos diferentes descritores de dor usados pelos pacientes para descrever tanto a dor clínica aguda quanto a crônica ${ }^{7,8}$.

De fato, aparentemente, todos concordam que a dor constitui-se numa experiência subjetiva, pessoal e multidimensional que envolve dimensões psicológicas, 
comportamentais, afetivas, cognitivas e sensoriais. Por ser um fenômeno multifacetado, a dor é também afetada pela experiência passada e pela cultura. Assim, medir a intensidade da dor é de suma importância para os pesquisadores e para os clínicos, pois a sua mensuração é essencial para a avaliação dos métodos que a controlam. A avaliação/mensuração da dor é um pré-requisito fundamental para o seu tratamento e manipulação eficazes ${ }^{9-11}$. Visto que a dor é uma experiência genuinamente subjetiva, apenas aqueles que a sentem podem determinar sua severidade e também a adequação de seu alívio. Em outras palavras, apenas a perspectiva do paciente é a correta e, portanto, suas autoavaliações são as mais acuradas e as mais confiáveis $^{12,13}$. Por consequência, é razoável questionar como tal fenômeno pode ser avaliado ou mensurado. Pelo fato da dor ser uma experiência subjetiva é possível somente avaliá-la ou mensurá-la por meio das variadas respostas ou reações manifestadas pelas pessoas que a vivenciam. Entretanto, qual aspecto da dor deve ser primariamente considerado, avaliado ou mensurado? Sua intensidade ou os seus variados componentes hedônicos? Para isso, torna-se essencial definir ou esclarecer o que significam os termos avaliação e mensuração no contexto da dor.

\section{AVALIAÇÃO VERSUS MENSURAÇÃO}

Os termos avaliação e mensuração da dor são frequentemente usados como sinônimos, mas muitos autores entendem que é importante distingui- $\operatorname{los}^{14}$. A mensuração é uma tentativa de quantificar a experiência individual da sensação de dor em comparação com outros indivíduos experienciando a mesma sensação, enquanto avaliação é parte de um processo global e é muito mais ampla que uma simples mensuração.

Certamente os mesmos instrumentos escalares podem ser utilizados tanto para a avaliação quanto para a mensuração, mas os usos das informações geradas diferem. As escalas de mensuração são essenciais na pesquisa, mas elas são também úteis na prática clínica onde esteja envolvida uma grande equipe.

Categoricamente afirmam que apenas uma simples medida da dor não é suficiente quando são consideradas as múltiplas facetas da experiência da dor ${ }^{15}$. Do mesmo modo, sugere que a mensuração envolve quantificar a dor, enquanto avaliação envolve uma compreensão global da experiência dor ${ }^{9}$. Em resumo, perguntar apenas se um paciente tem dor não é sufi- ciente. Uma avaliação global considerando todas as múltiplas dimensões da sensação de dor é imprescindível, especialmente em contextos clínicos. Mas é a sensação de dor mensurável? Certamente é.

\section{A mensuração da sensação de dor}

A dor tem sido considerada uma experiência sensorial e emocional subjetiva desagradável, difícil de quantificar e qualificar. É um fenômeno complexo, derivado de estímulos sensoriais ou de lesões neurológicas, e que pode ser modificado pela memória, pelas expectativas e pelas emoções dos indivíduos. Além disso, a dor pode ser influenciada por uma variedade de outros fatores, incluindo as habilidades para manuseá-la e controlá-la, os sinais vitais, a história médica e cirúrgica, as condições socioeconômicas, o contexto cultural, o sexo e as habilidades intelectuais ou cognitivas.

A dor é usualmente associada com lesão ou a um processo patofisiológico que causa uma experiência desconfortável e desagradável sendo, geralmente, descrita em tais termos. Por ser assim definida, ela constitui-se uma experiência multidimensional e, portanto, sua avaliação engloba a consideração de inúmeros domínios, incluindo o fisiológico, o sensorial, o afetivo, o cognitivo, o comportamental e o sociocultural. Em outras palavras, a dor afeta o corpo e a mente, e sua complexidade torna-a difícil de ser mensurada.

Embora não haja qualquer marcador biológico da dor, a descrição individual e o autorregistro geralmente fornecem evidências acuradas, fidedignas e suficientes para detectar a presença e a intensidade da dor. De fato, não há qualquer marcador biológico disponível até o presente momento para indicar a presença ou o grau da dor, exceto os indicadores manifestados por aqueles vivenciando dor.

De acordo com o Instituto Nacional de Saúde (NIH), dos Estados Unidos, a autoavaliação é o "indicador mais confiável da existência e da intensidade da dor". As medidas da dor que podem ser quantificadas incluem a intensidade, a localização a distribuição, a duração e periodicidade, a qualidade, sinais associados e sintomas, o impacto e o significado pessoal ${ }^{2,16,17}$. O instrumento ideal para avaliação da dor, portanto, deve incluir a identificação da presença de dor bem como o progresso da dor com o tempo ou em função do tratamento. Este instrumento deveria também ser aplicável a qualquer indivíduo, independente das características fisiológicas, emocionais ou culturais.

Também, para manuseio e controle eficientes da dor, torna-se necessário avaliá-la de forma regular e consis- 
tentemente, tal como é usualmente feito com os outros sinais vitais. Porém, temperatura, pulso, respiração e pressão sanguínea podem ser objetivamente mensuradas, enquanto a sensação de dor considerada como o "quinto sinal vital" é inerentemente subjetiva.

Todavia, não é suficiente perguntar, "quão intensa é sua dor numa escala de zero a 10 ?". O clínico deve avaliar cuidadosamente os aspectos multidimensionais do fenômeno da dor para desenvolver um compreensivo programa de atendimento ao paciente, pois uma avaliação precisa e confiável da dor é crucial para um planejamento eficiente para o seu tratamento. É impossível manipular um problema clínico sem ter uma medida sobre a qual basear o tratamento. Sem mensuração, não se pode determinar se o tratamento é necessário, se o tratamento prescrito é eficiente, ou quando interrompê-lo. Não se pode determinar se os riscos de um tratamento superam os malefícios causados pela condição física e, além disso, não se pode escolher logicamente entre os diferentes tipos de tratamentos. A mensuração habilita-nos a examinar a natureza, as origens e os correlatos da dor. Em algumas circunstâncias, medidas grosseiras, como "dor presente" ou "dor ausente", são necessárias para intervenções clínicas, mas para um completo entendimento do fenômeno e para avaliar a eficácia das intervenções tornam-se necessárias medidas sofisticadas da intensidade e da dimensão afetiva da dor.

A avaliação incorreta da dor é a causa principal do manejo inapropriado da dor. O motivo mais comum para o tratamento incorreto da dor nos hospitais norte-americanos tem sido o fracasso dos clínicos em avaliarem a dor e o seu alívio. Para minimizar isso tem havido inúmeros esforços e campanhas para elevar a consciência dos clínicos sobre a importância da avaliação e mensuração da dor. Como consequência direta desses esforços, em 1996, A Sociedade Americana de Dor (American Pain Society) introduziu o conceito "dor como o quinto sinal vital”. Esta iniciativa enfatiza que a avaliação da dor é tão importante quanto a avaliação dos outros quatro sinais vitais padrão e que os clínicos necessitam agir quando os pacientes registram dor. Também reconhecendo a importância da avaliação da dor, outras sociedades norte-americanas ligadas à saúde, reconheceram o valor de tal abordagem e incluiu a dor como o quinto sinal vital em suas campanhas nacionais acerca do seu manejo e o seu controle. Da mesma forma, em 14 de junho de 2003, a Direção Geral de Saúde, em Portugal, estabeleceu como norma de boa prática nos serviços prestadores de cuidados de saúde, o "registro sistemático da intensidade da dor", elevando-a a dignidade de $5^{\circ}$ quinto sinal vital - juntando-se a frequência respiratória, pressão arterial, frequência cardíaca e temperatura.

\section{As dimensões da sensação de dor}

Antes do século XIX, a dor era definida como um fenômeno da mente e poucas tentativas foram feitas para tentar explicá-la em termos de seus mecanismos anatômicos e fisiológicos. Com a evolução do pensamento científico houve grandes progressos no conhecimento da anatomia e fisiologia do corpo humano e, por consequência, uma escola de pensamento emergiu, a qual considerava a dor como um sistema sensorial humano, similar à audição ou visão, com os seus próprios substratos neurológicos. A transmissão da informação da dor foi considerada como estando ao longo de uma via direta, indo desde os receptores periféricos até o centro de dor no cérebro. Esta visão reducionista da experiência da dor supunha que a dor era uma sensação específica e que sua intensidade era proporcional à extensão da lesão dos tecidos. Este "modelo nociceptivo" da experiência da dor ainda permanece como padrão em muitas intervenções clínicas.

Porém, recentemente, o conceito de dor tem mudado de um simples sinal neurofisiológico para um fenômeno psicofisiológico complexo, com muitos correlatos inter-relacionados, ainda que pouco entendidos. Esta mudança ocorreu, em parte, por causa das recentes pesquisas demonstrando que a gravidade da dor registrada pode estar relacionada a sintomas fisiológicos específicos, combinados com o efeito de uma ou mais variáveis psicológicas. Fatores culturais, influências sociais moduladoras, assim como, fatores de personalidade e comportamentos instrumentais podem influenciar o registro da dor. Desde que a experiência da dor possa ser influenciada por uma composição destes fatores, torna-se evidente que qualquer tentativa séria para avaliá-la deve incluir medidas que sejam sensitivas a tal modulação. Todavia, é surpreendente que o progressivo entendimento dos fatores psicológicos, que modulam a sensação de dor, não tenha apresentado grande influência nos procedimentos utilizados para avaliar a dor clínica. Em muitos contextos, como mencionado, a dor ainda é mensurada ao longo de uma dimensão unitária da intensidade. A mensuração da dor, baseada exclusivamente nessa dimensão, fracassa em reconhecer as características reativas (emocionais) e sensoriais (sentimentos) da dor.

Claramente, não há uma correspondência um a um entre lesão nos tecidos e sensação de dor. A dor é uma experiência perceptual, subjetiva, e a característica que 
a diferencia de uma simples sensação é exatamente sua qualidade afetiva. Assim, a dor parece ter duas propriedades genuínas: (a) uma sensação corporal e (b) um efeito afetivo de natureza aversiva. Por sua vez, cada uma dessas propriedades pode ser caracterizada ao longo de outras dimensões: sensações podem ser externas (por exemplo: pressão, temperatura) ou internas (dolorido, queimação), e o afeto pode pertencer ao nível de excitação ou a emoções qualitativamente diferentes (por exemplo: medo, raiva, tristeza). Há também fenômenos associados, tais como antecedentes neurais e conseqüentes comportamentais ${ }^{18}$. Mas, categoricamente, a dor é vivenciada em um nível físico e em um nível afetivo. Como enfatizada pela Associação Internacional para o Estudo da Dor ${ }^{19}$, "a dor é inquestionavelmente uma sensação numa parte ou partes do corpo, mas ela é também sempre desagradável e, portanto, é sempre uma experiência emocional" (S 217).

Entretanto, apesar do aparente consenso de que a dor tem componentes sensoriais e afetivos, nem todos estão convencidos da separação entre estes componentes. De um lado, alguns autore ${ }^{20}$ argumentam que é impossível, introspectivamente, separar sensação, percepção e cognição, e que seria mais apropriado falar de causas contribuintes em lugar de componentes separados da dor. Para estes autores, a ideia dos componentes de sensação e reação como partes distinguíveis da experiência não é bem corroborada e, também, não possui uma justificativa convincente, podendo, portanto, pode ser provavelmente descartada. De outro lado, outros autores ${ }^{21}$ propõem que dor e emoção são processados separadamente e em paralelo. Para eles, os eventos sensoriais e a informação afetiva são processados quase simultaneamente, e a separação dos componentes sensoriais e afetivos aparece no início da codificação do estímulo, de acordo com o tipo de especialização do receptor. Porém, muitas dessas abstrações não são facilmente testáveis, embora algumas delas possam ser consistentes com evidências neurofisiológicas.

Assim, a comprovação da questão da separação entre dimensão sensorial e dimensão afetiva da dor deve necessariamente ser baseada em evidências empíricas, e recentemente há vários estudos que a sustentam. Tais estudos, realizados com este propósito, podem ser agrupados em quatro grandes grupos: (1) análise estatística multivariada, (2) aplicações da Teoria da Detecção de Sinal (TDS), (3) escalonamentos psicofísicos unidimensionais e (4) imageamento cerebral para identificar um substrato neural comum da dor.

\section{Análise estatística multivariada}

A estatística multivariada tem sido introduzida com o propósito de verificar se de fato os descritores de dor se enquadram ao longo de dimensões rotuladas como sensorial e afetiva. A metodologia tem envolvido principalmente a análise fatorial (Factor Analysis - FA) e em menor extensão a análise multidimensional (Multidimensional Scaling - MDS) e a análise de conglomerados (Cluster Analysis). Comum, a todos estes procedimentos, é a análise simultânea de um número de variáveis sem designá-las como independente ou dependente, com o propósito de descobrir a estrutura latente destas variáveis. Tomados em conjunto, estes procedimentos revelam indicações que permitem supor claramente a existência da separação dos componentes sensorial e afetivo da dor. Há, porém, dois grandes problemas metodológicos nestes procedimentos. Em primeiro lugar, as amostras têm sido sempre pequenas. Para gerarem dados confiáveis, estes procedimentos requerem amostras com, pelo menos, 200 sujeitos, ou uma amostra pelo menos cinco vezes maior que o número de variáveis englobadas pelos fatores esperados, além de uma rotulação criteriosa dos fatores e a incorporação de uma série sistemática de estudos em que a estrutura dos fatores é progressivamente refinada. Em segundo lugar, há limitações inerentes na análise multivariada que usualmente impõem constrangimentos no problema focalizado. Por exemplo, os tipos de estímulos selecionados e a tarefa designada influenciam as respostas dos sujeitos.

\section{Aplicações da Teoria da Detecção de Sinal (TDS)}

Em contraste com a análise estatística multivariada do vocabulário da dor, a teoria da detecção de sinal tem frequentemente permitido a demonstração experimental dos componentes da dor. A identificação desses componentes também tem sido problemática. A interpretação de d' e $\beta$ em termos unitários é aberta a desafios. $\mathrm{O}$ critério de respostas $\beta$, representa o grau de prontidão para registrar a dor, e esta razão de verossimilhança é um produto de multifacetadas variáveis psicológicas (tais como atitude, recompensa, características da demanda e personalidade) da qual emoção é um dos ingredientes. Algumas intervenções, como manipulações médicas e psicológicas, envolvendo o uso de tranquilizantes e expectativas (através da sugestão ou placebo), claramente identificam um componente afetivo como distinto das propriedades sensoriais da dor. Num sentido estrito, a TDS faz uma substancial distinção entre os constituintes sensorial e psicológico da dor. 
Outra preocupação é com a caracterização do d'. Este índice não é apenas um indicador da sensibilidade da presença ou ausência do estímulo (isto é, a detecção do sinal). Na pesquisa de dor, entretanto, ele representa a discriminabilidade entre os vários estímulos adjacentes que causam dor, considerando que é virtualmente impossível apresentar um estímulo de intensidade zero sem evocar sensações de tato ou de temperatura, como ocorre nesse caso. Por consequência, d' mede a sensibilidade diferencial em vez da sensibilidade absoluta à dor. A conceituação de d’ tem sido revisada, pois há demonstração de que este parâmetro pode também ser influenciado por variáveis cognitivas tais como déficits de memória e perseverança na resposta.

Assim, segue-se que d' e $\beta$ não são medidas puras das respostas sensoriais e afetivas, respectivamente, porque eles são influenciados também por fatores cognitivos. Além disso, ambos os índices podem não ser abertos à avaliação independente. A sensação pode influenciar o critério de resposta e vice-versa. E como ocorre com os procedimentos da análise estatística multivariada, apesar da separação estatística com o uso da TDS, os dois componentes da dor podem ser funcionalmente relacionados.

A TDS tem sido usada em vários estudos ${ }^{22-24}$ para examinar a influência do placebo, do óxido nítrico, do diazepam (um bloqueador neuromuscular menor), da morfina (um narcótico analgésico), da acupuntura e da estimulação elétrica transcutânea no nervo. Dor, nestes casos, foi evocada por estimulação elétrica da polpa dentária ou por calor aplicado à pele. Os resultados destes estudos foram consistentes com uma interpretação de d' como sensibilidade à dor e o critério de resposta $(\beta)$ como um viés de resposta. A administração do placebo resultou em apenas uma variação no critério de resposta sem uma mudança em d', enquanto as intervenções ativas reduziram d' com efeitos variáveis sobre o critério de resposta. Estes resultados têm despertado novos interesses para esta técnica e reavivado a atenção sobre a avaliação da analgesia e dos vieses de resposta ${ }^{25-27}$.

\section{Escalonamentos psicofísicos}

Talvez, a metodologia psicofísica mais comumente usada para a mensuração da sensação de dor seja as escalas de estimação unidimensionais. Para medir o componente sensorial em relação ao componente afetivo da dor, esta abordagem tem feito uso de escalas pareadas, onde uma dada escala é usada para quantificar cada componente em separado. Se estimativas diferenciais são obtidas em cada escala, corrobora-se a distinção entre as dimensões afetivo-sensorial. As escalas de categorias, as escalas analógicas visuais e as escalas de razão têm sido frequentemente utilizadas com este propósito. Todavia, cada uma delas apresenta problemas metodológicos que devem ser considerados.

Com o uso das escalas de categorias há dois problemas centrais. Primeiro, pelo fato do número de categorias com as quais os estímulos são julgados serem fixos, o método introduz sérios vieses. Por isso, as escalas de categorias são especialmente sensíveis aos efeitos de contexto, tais como, a amplitude das categorias e a frequência dos estímulos. No caso da mensuração da dor, uma maior fonte de erro tem sido o constrangimento causado ao examinando pela imposição de uma âncora ou limite superior, no fim do contínuo de dor, isto é, da escala de mensuração da dor. Segundo, as escalas de categorias não permitem afirmações sobre a razão de diferenças entre as medidas de dor obtidas. Tem significado afirmar que uma medida é maior do que outra ou subtrair uma da outra, mas não é possível deduzir quantas vezes uma medida é maior ou menor que outra. Num esforço para minimizar algumas das limitações das escalas de categorias, as escalas analógicas visuais têm atraído considerável interesse na mensuração da dor. Devido às suas características psicométricas e facilidade de uso pela maioria das pessoas, estas escalas têm sido usadas para diferenciar dimensões sensoriais e afetivas da dor. Os dados usualmente têm mostrado estimativas consistentemente mais altas para a sensação do que para o afeto em condições em que a dor foi experimentalmente induzida. Adicionalmente, às estimativas obtidas nessas escalas ajustam-se perfeitamente á funções de potência, com um diferente expoente para a dimensão sensorial e a dimensão afetiva. Este padrão de resultados tem sido tomado como forte suporte de que estas escalas podem separar as duas dimensões da experiência da dor.

Porém, dois problemas metodológicos devem ser apontados. Primeiro, as medidas obtidas com as escalas analógicas estão sujeito à variabilidade dependendo do comprimento da escala e do modo como as extremidades da escala são rotuladas. Ademais, as instruções podem influenciar como os sujeitos respondem às solicitações para emitir respostas diferenciais às dimensões sensoriais e afetivas. Segundo, embora as estimativas analógicas sejam um caso especial do método de emparelhamento intermodal entre modalidades diferentes, sua adaptação para a mensuração da dor contradiz as propriedades de razão. As estimativas analógicas estão 
ancoradas no topo, diferente das escalas de razão. Além disso, ela carrega um elemento das escalas de categorias porque descritores são comumente usados para demarcar vários pontos na escala e estes podem distorcer a distribuição das estimativas.

A escala de razão constitui o nível mais alto de mensuração. Ela requer um ponto zero não-arbitrário ou origem e intervalos iguais entre as escalas. Tal escala é comumente obtida pelas estimativas de magnitude (Magnitude Estimation - ME) em que os sujeitos assinalam um número facilmente relembrado, por exemplo, 10, a um estímulo padrão e, então, assinalam números aos estímulos subsequentes, de modo que a razão entre os números assinalados e o número 10 reflita a razão entre a sensação produzida pelo estímulo variável e a sensação produzida pelo estímulo padrão; alternativamente, os sujeitos podem ajustar o comprimento de uma linha ou a força dinamométrica para emparelhar mudanças proporcionais na magnitude do estímulo, um procedimento denominado de emparelhamento intermodal (Cross-Modality Matching- CMM). Este tem sido o método dominante utilizado com o propósito de derivar escalas de razão para as dimensões afetivas e sensoriais da dor.

Os estudos que utilizaram estes métodos psicofísicos têm revelado que as estimativas de magnitude, assim como os emparelhamentos intermodais dos descritores de dor têm produzido uma amplitude maior para as estimativas da dimensão sensorial do que aquelas para a dimensão afetiva. Este padrão de resultados é tomado como suporte da idéia de que há um contínuo para a dor sensorial e um contínuo separado para a dor afetiva.

Interessante notar que sob intervenção farmacológica, as estimativas de razão dos dois componentes da dor revelam alteração seletiva. O fentanil que é um opioide reduz a dor sensorial, mas não afeta a sua contraparte afetiva. De outro lado, o diazepam reduz a afetiva, mas não a sensorial. Isto vai de encontro com os efeitos documentados destes fármacos: os opioides tendem a exercer um efeito analgésico, mas os tranqüilizantes menores têm seu efeito alterando o ânimo ${ }^{28,29}$.

Tem havido também vigorosos debates sobre se as estimativas obtidas com estes tipos de escalonamentos se conformam às escalas de razão. Por exemplo, tem rejeitado a suposição das propriedades de razão destas escalas ${ }^{30}$. Ele apontou que a exigência de um nível de mensuração de razão com um ponto zero não-arbitrário, racional, raramente é satisfeito, até mesmo nas ciências naturais e, provavelmente, é inatingível em psicologia. Apontou, também, que os sujeitos poderem fazer ajustamentos intermodais proporcionais à razão das diferenças entre dois estímulos é também uma fonte de dúvida. Replicaram a estas críticas fazendo menção a uma vasta literatura confirmando as escalas de razão obtidas através do emparelhamento intermodal ${ }^{28}$. Afirmaram que, se estes métodos produzem medidas em nível de razão das dimensões afetiva e sensorial da dor, é um assunto discutível ${ }^{29}$. De fato, a constatação da existência de funções psicofísicas separadas para dor sensorial e dor afetiva é uma evidência insuficiente para a separação dos dois componentes. Porém, o mais importante é a divergência entre as duas funções ou uma diferença entre suas inclinações, e ambas têm sido invariavelmente encontradas em vários estudos, explorando tanto a dor experimental quanto a dor clínica, usando o método do emparelhamento intermodal.

\section{Imageamento cerebral}

O uso das técnicas de imageamento cerebral como, por exemplo, Tomografia por Emissão de Pósitrons (PET), Imagens por Ressonância Magnética Funcional (fMRI) em humanos, tem permitido aos pesquisadores investigarem as bases neurais da experiência sensorial complexa e emocional da dor. Examinando a percepção, concomitantemente com a atividade neural em humanos conscientes, os estudos têm permitido revelar como o cérebro processa a complexa sensação da dor. De fato, uma combinação da mensuração de múltiplos indicadores perceptuais com o imageamento cerebral permite aos pesquisadores examinarem a determinação neural de diferentes aspectos da experiência da dor. Usando análises correlacionais, diferentes substratos neurais das dimensões sensorial e afetiva da experiência da dor têm sido comparados e elas têm revelado que estes dois construtos (sensorial e afetivo) não são isomórficos. O componente afetivo da dor, isto é, o quão desagradável é a experiência da dor, é altamente influenciado pela intensidade da sensação da dor. Num esforço para dissociar as dimensões sensoriais e afetivas da dor, os pesquisadores têm também feito uso das sugestões hipnóticas em sujeitos experimentais para seletivamente alterar a intensidade percebida ou o desprazer de um estímulo doloroso (por exemplo, temperatura) apresentado na mão do sujeito ${ }^{31}$. A partir de análises correlacionais entre as percepções dos sujeitos e as mudanças no fluxo sanguíneo cerebral evocado pela dor, os pesquisadores revelaram que, a ativação evocada da dor no córtex somatossensorial primário (S1) foi mais altamente relacionada à dimensão sensorial da dor, enquanto que o córtex anterior cingulado (ACC) 
foi mais bem relacionado à dimensão afetiva $a^{32,33}$.

Tem afirmado que os estudos sobre o imageamento cerebral em humanos vivenciando dor indicam que há uma rede de estruturas corticais e subcorticais que estão subjacentes a esta experiência, seja a dor originada de lesão tecidual periférica ou a partir de anormalidades do sistema nervoso central ${ }^{33}$.

Pelo fato da experiência sensorial e emocional associada com a dor variar amplamente entre indivíduos, bem como dentro do mesmo indivíduo em diferentes momentos e em diferentes contextos, torna-se obviamente importante combinar rigorosos métodos quantitativos com as técnicas de imageamento cerebral. As dissimilaridades vivenciadas são refletidas em vários padrões de ativação neural observada em diferentes estudos experimentais. Todavia, apesar dessas diferenças, muitos aspectos comuns emergem destes estudos, incluindo a ativação de regiões sensoriais, tais como o córtex somatossensorial primário e o córtex somatossensorial secundário (S2) e áreas límbicas, tais como o córtex anterior cingulado e o córtex insular (IC). O mais importante é que o grau de ativação destas regiões é dependente de fatores cognitivos, tais como o estado de atenção, que altera a percepção de dor. Portanto, quando um paciente vivencia dor, independente de sua origem, pelo menos alguns componentes desta rede cortical são prováveis de serem ativadas e podem ter distintos determinantes neurofisiológicos ${ }^{34,35}$.

\section{Como as dimensões da dor são mensuradas?}

A experiência da dor pode ser mensurada considerando duas suposições básicas. Primeira, que a dor é uma dimensão univariável e unitária, isto é, ela é considerada como uma simples dimensão variando apenas em magnitude. Segunda, a percepção da dor é claramente uma rica e multidimensional experiência, variando na qualidade sensorial, na intensidade sensorial e em características afetivo-motivacionais. No primeiro caso a dor usualmente é avaliada por meio de escalas unidimensionais e, no segundo caso, por meio de escalas/ questionários multidimensionais. Nas escalas unidimensionais, os estímulos podem ter muitos atributos, mas há apenas uma dimensão psicológica de interesse. Portanto, usualmente elas avaliam uma simples e única dimensão da dor, isto é, a magnitude da intensidade da dor comumente registrada pelo paciente. Embora úteis para avaliarem a dor aguda de etiologia bem definida (por exemplo, a dor no pós-operatório), as escalas de estimação da intensidade podem simplificar a avaliação de algum tipo de dor. Para evitar isso muitos clíni- cos recomendam o uso das escalas multidimensionais na avaliação de dor complexa ou persistente, pois entendem que a dor é mais bem avaliada quando muitos indicadores são utilizados.

Com as escalas multidimensionais, os estímulos são representados por valores psicológicos sob mais que uma dimensão ou atributo. Desta forma, múltiplos indicadores das diferentes dimensões da experiência da dor, tanto de suas dimensões primárias, a intensidade e o desprazer, quanto de suas múltiplas dimensões, tais como localização, duração, intensidade e qualidade, e que fornecem o máximo de informação possível sobre as respostas individuais da dor e suas inúmeras interações, devem ser incluídos em qualquer escala multidimensional. Na realidade, as escalas unidimensionais ou bidimensionais, de estimação da magnitude da intensidade ou do desprazer da dor, podem ser classificadas como técnicas restritas porque o número e a qualidade das dimensões são pré-determinadas antes que a mensuração seja feita. As escalas multidimensionais não-restritas permitem aos pacientes determinarem o número e o tipo de dimensões e são, portanto, supostas ser menos enviesadas, melhor representando a realidade da experiência da dor. Entretanto, esta diferenciação não é totalmente clara porque o objetivo, mesmo das escalas não restritivas, é descobrir as dimensões reais da dor e, portanto, conduzir à mensuração restritiva. Inversamente, o sistema de dimensões sensoriais e afetivas foi, em parte, descoberto através da categorização empírica dos registros de dor ${ }^{17}$.

\section{Tipos de indicadores da sensação de dor}

Há três grandes categorias nas quais podem ser agrupados os indicadores da sensação de dor: (1) indicadores obtidos através da autoavaliação (autorregistros) da dor, (2) indicadores observáveis (comportamentais) da dor e (3) indicadores fisiológicos (alterações biológicas) da dor. Às vezes, estes indicadores são agrupados em apenas duas amplas categorias: indicadores fisiológicos e indicadores psicofísicos. A figura 1 mostra, esquematicamente, um modelo de avaliação e mensuração da sensação de dor, considerando estas duas categorias. Dentre as medidas fisiológicas, ditas objetivas pode-se destacar a resposta galvânica da pele, os batimentos cardíacos, os potenciais evocados, a dilatação da pupila, a pressão sanguínea, a sudação palmar, a saturação de oxigênio, a pressão intracraniana, o fluxo sanguíneo na pele e imagens das diferentes áreas do cérebro por ressonância magnética funcional (fMRI) ou por Tomografia por Emissão de Pósitrons (PET). Dentre as medi- 
das psicofísicas, ditas subjetivas destacam-se as escalas unidimensionais e multidimensionais dirigidas aos atributos sensorial, hedônico e avaliativo da sensação de dor. Importante no modelo é a inclusão de variáveis que modulam a percepção, a sensação, a intensidade e a qualidade da dor vivenciada e, então, registrada. Variáveis atentivas, mnemônicas, cognitivas e motivacionais podem diretamente afetar a dimensão sensorial, bem como o caráter hedônico da dor clínica e mesmo da dor experimental percebida. De fato, provavelmente, a variável psicológica mais estudada que modifica a experiência da dor, é o estado atentivo.

Um grande número de estudos registra que a dor é percebida de forma menos intensa quando os indivíduos são distraídos da dor. A distração é manipulada fazendo-se com que o paciente atenda a outra modalidade sensorial, como a visual, a auditiva ou a um estímulo tátil, conduzindo a uma modulação sensorial intermodal similar àquela observada em outras modalidades ${ }^{36,37}$. Interessante observar também que alguns autores têm usado técnicas hipnóticas ${ }^{31}$ e a técnica de realidade virtual imersiva para controlar e/ou modular a dor ${ }^{38}$. Além disso, variáveis culturais, raciais e de sexo, também podem afetar a percepção e a experiência da dor ${ }^{39,40}$.

\section{Indicadores por autorregistros da sensação de dor}

A pessoa se queixando de dor fornece informações para completar a medida da dor. As autoavaliações são usadas de várias formas. Elas frequentemente envolvem a estimação através de alguma escala métrica. Um clínico pode solicitar ao paciente para estimar a dor mais intensa (a pior dor), a menor dor e a dor moderada percebida na última semana. Diários como papéis e lápis ou eletrônicos, constituem outros modos de obter uma prospectiva, visão subjetiva da dor de um paciente, se a dor é persistente ou crônica. É um modo muito útil de medir o impacto que a dor causa na vida funcional do paciente.
Os diários podem ser relativamente estruturados contendo a informação necessária para registro preparada num formato que é completada em intervalos regulares e contínuos como, por exemplo, o registro contínuo ou em intervalos fixos da dor percebida durante um período de tempo. Estimativas da intensidade da dor, níveis de repouso e de atividades, e estados afetivos, emocionais e sentimentos atuais podem também ser registrados.

$\mathrm{O}$ autorregistro é considerado um instrumento padrão da mensuração da dor porque ele é consistente com a própria definição da dor. Dor é uma experiência subjetiva. Mas, o dilema das medidas derivadas da autoavaliação, reside exatamente, na sua natureza subjetiva. Elas são baseadas na percepção do paciente de sua dor e esta percepção pode ser influenciada por outros fatores. Por exemplo, que certeza o clínico tem de que o paciente está dando respostas honestas, confiáveis, ou que estas respostas não tenham sido enviesadas por outros fatores não inerentes à percepção de dor.

Tem havido uma controvérsia sobre a validade dos dados obtidos através da autoavaliação; alguns trabalhos revelam que o nível de dor registrado pelos pacientes com dor crônica não foi relacionado com os seus respectivos registros de incapacidade física ${ }^{41}$. $\mathrm{O}$ dilema aqui reside no fato de que é esperado que o grau de incapacidade física seja proporcionalmente relacionado à severidade da dor. Quando ambos não se relacionam deste modo, argumenta-se que o autorregistro da dor do paciente é exagerado e, portanto, inválido. Certamente isto é o esperado, mas o desempenho físico atual e o nível de desempenho físico percebido podem ser dois construtos inteiramente diferentes, cada um dos quais dando informação clínica válida sobre um paciente com dor clínica. Finalmente, as medidas do autorregistro baseiam-se na habilidade de uma pessoa se comunicar sobre a dor. O autorregistro não é possível com infantes, crianças jovens, ou com pessoas com necessidades especiais que dificultam a comunicação.

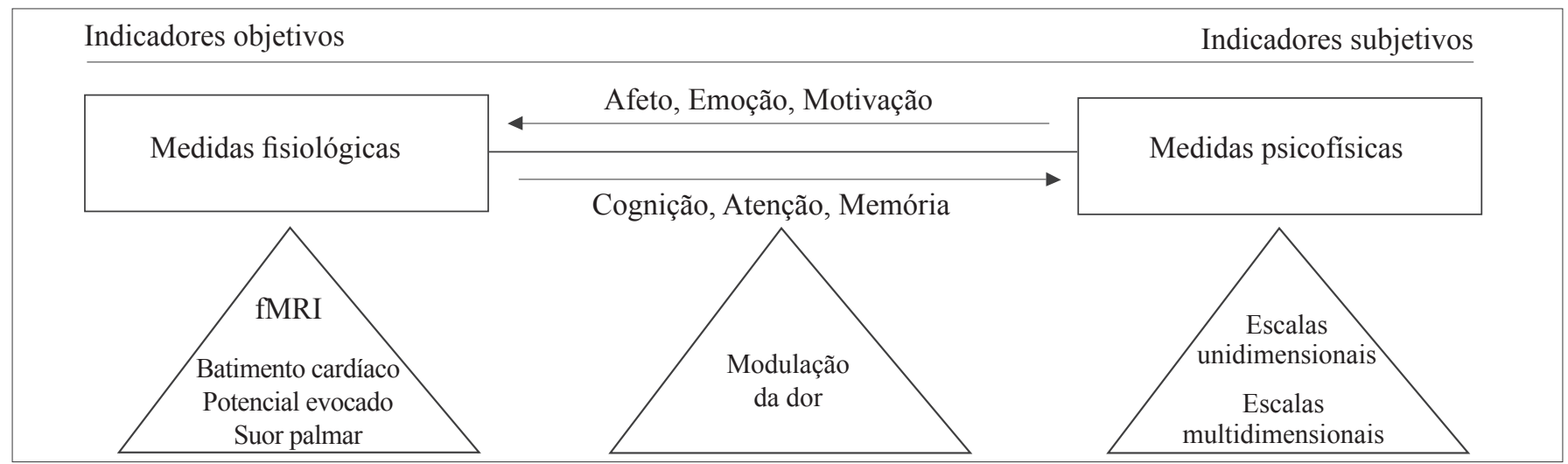

Figura 1 - Interação entre os diferentes indicadores da sensação de dor 


\section{Indicadores comportamentais da sensação de dor}

Os indicadores de comportamentos observáveis constituem outro conjunto de reações capturadas pelas escalas de mensuração da dor. Estes indicadores, usualmente, feitos por um clínico, ou por alguém muito familiar ao paciente, consistem no registro de algum comportamento observável associado à experiência da dor, usualmente referente ao comportamento ou desempenho físico. Exemplos são: o choro; as expressões faciais tais como, sobrancelhas franzidas, olhos cerrados, afundamento da prega nasolabial, lábios abertos, boca alargada verticalmente e língua tensa e côncava; os movimentos físico-corporais dos braços e das pernas, o tônus muscular, a agitação, a excitabilidade, a consolabilidade, etc. São frequentemente utilizados para avaliar a dor em recém-nascidos, crianças pré-escolares, idosos e em pessoas portadores de deficiência física ou cognitiva. Além disso, estes indicadores podem ser úteis para corroborar os autorregistros dados pelo paciente. Eles são também úteis para identificar outras áreas preocupantes, particularmente a mensuração do funcionamento físico e os fatores ergonômicos que podem exacerbar ou causar dor relacionada ao trabalho. Os componentes subjetivos podem ajudar em determinar qual tipo de programa de tratamento é mais apropriado para qual tipo de paciente com dor ${ }^{42}$. Não obstante, as medidas comportamentais, como uma técnica, por exemplo, podem ser relativamente custosas, pois requerem muito tempo de observação, além de treino intensivo sobre as técnicas de observação. Elas podem também ser menos sensíveis aos componentes afetivos e subjetivos da experiência da dor.

Em pesquisa, as medidas comportamentais têm-se mostrado mais acuradas para dor aguda desde que o comportamento de dor tende a se habituar quando a dor torna-se mais crônica. Entretanto, também, não há qualquer comportamento que seja um indicador de dor. Compressão do abdome, por exemplo, pode ser devido à dor, mas pode também ser um espasmo de náusea. Para conhecer o que cada comportamento significa, deve-se perguntar ao paciente e isto nada mais é que o próprio autorregistro.

Por último, os indicadores observáveis aparentemente constituem uma medida mais objetiva da dor do paciente, mas elas podem refletir a subjetividade das mensurações feitas pelo clínico, ou por alguém conhecido do paciente, da dor do paciente. Aqueles que fazem tais mensurações devem ser necessariamente treinados a identificar e a registrar quais comportamentos estão associados ou não à dor.
Indicadores fisiológicos/biológicos da sensação de dor A terceira categoria de indicadores úteis para a mensuração da sensação de dor é fisiológica. A dor causa mudanças biológicas na frequência cardíaca, respiração, transpiração, tensão muscular, pressão arterial e outras mudanças associadas com o estresse, a agonia e a aflição da resposta ${ }^{43}$. Estas mudanças biológicas podem ser usadas como uma medida indireta da dor aguda, mas a resposta biológica à dor aguda pode estabilizar-se ao longo do tempo como tentativas do corpo para recuperar sua homeostase. Por exemplo, a respiração e os batimentos cardíacos podem mostrar pequenas alterações no início de uma enxaqueca, principalmente se o início for relativamente repentino e severo, mas com o decorrer do tempo estas oscilações provavelmente retornam às taxas anteriores ainda que possa persistir a enxaqueca. Medidas desses indicadores fisiológicos são úteis em situações onde as medidas comportamentais observáveis são mais difíceis. Por exemplo, medidas comportamentais podem ser usadas para medir a dor em infantes, mas as medidas fisiológicas têm fornecido importante informação sobre a dor pós-cirúrgica em recém-nascidos ${ }^{18,44}$. Em resumo, as medidas de autorregistro são consideradas o padrão-ouro na mensuração ou avaliação da dor. Afinal, somente o paciente conhece exatamente quão intensa e o quão perturbadora é a dor que ele sente. As medidas feitas por outros, o clínico ou alguém próximo do paciente como o conjugue, são úteis, mas estas mensurações são indiretas. É ainda muito importante notar que todas estas três categorias de medidas têm algum grau de erro. Elas fornecem uma parte da configuração geral (da gestalt) da experiência de dor do paciente, mas elas não têm $100 \%$ de precisão.

Nos capítulos seguintes vamos discutir os vários procedimentos e/ou técnicas que podem ser usadas para obter uma descrição mais completa da sensação de dor, clínica e experimental ou induzida. As escalas unidimensionais e as escalas multidimensionais mais comumente usadas para mensurar e avaliar a sensação de dor, tanto em adultos quanto em crianças, serão descritas, incluindo suas propriedades psicométricas quando aferidas. Importante, todavia, destacar novamente que as medidas que descrevem a dor são, por natureza, autoavaliativas. Elas são tipicamente na forma de questionários, inventários, formulários, escalas de estimação, escalas analógicas visuais e desenhos. Lembrando ainda que a sensação de dor possa ser descrita em termos de sua intensidade (isto é, o quão intensa ela é), de sua qualidade (isto é,o quão penetrante, difusa, 
dura, estonteante, etc. ela é), e sua localização espacial e temporal no corpo.

A descrição da dor de um paciente serve a vários propósitos. Uma descrição de linha de base permite comparações de mudanças. Idealmente, a dor poderia ser monitorada por algum tempo antes do início do tratamento, e então durante e no final do tratamento. As escalas resumidas, curtas, como as escalas de estimativas numéricas, têm sido usadas, diariamente, por até duas semanas em programas de acompanhamento de pacientes com dor crônica e os resultados, quando combinados, são usados para aumentar a fidedignidade da avaliação. A avaliação global, ainda que trabalhosa em tempo e esforço fornece uma linha de base para verdadeiramente comparar as mudanças que ocorrem após uma intervenção clínica e é mais do que necessária e desejável em muitos contextos clínicos. Há considerável evidência de que os autorregistros da intensidade da dor são tanto fidedignos quanto válidos ${ }^{45}$.

\section{REFERÊNCIAS}

1. Edwards RR, Sarlani E, Wesselmann U, et al. Quantitative assessment of experimental pain perception: multiple domains of clinical relevance. Pain 2005;114(3):315-9.

2. Gracely RH. Pain measurement. Acta Anaesthesiol Scand 1999;43(9):897-908.

3. Fillingim RB. Sex, gender and pain: women and men really are different. Curr Rev Pain 2000;4(1):24-30.

4. Livingston WK. What is pain? Scientific American 1953;196(1):59-69.

5. Melzack R. Neuropsychological basis of pain measurement. Ad Pain Res Ther 1984;6: 323-39.

6. Melzack R, Torgerson WS. On the language of pain. Anesthesiology 1971;34(1):50-9.

7. Verkes RJ, Van der Kloot WA, Van der Meij J. The perceived structure of 176 pain descriptive words. Pain 1989;38(2):219-29.

8. Radvila A, Adler RH, Galeazzi RL, et al. The development of a German language (Berne) pain questionnaire and its application in a situation causing acute pain. Pain 1987;28(2):185-95.

9. McGuire DB. Comprehensive and multidimensional assessment and measurement of pain. J Pain Symptom Manage 1992;7(5):312-9.

10. Vallerand AH. Measurement issues in the comprehensive assessment of cancer pain. Semin Oncol Nurs 1997;13(1):16-24.

11. Baillie L. A review of pain assessment tools. Nurs Stand 1996;7(1):25-9.
12. Twycross R. Pain relief in advanced cancer. London: Churchill Livingstone; 1994.

13. McCaffery M. Nursing the patient in pain. Philadelphia: JB Lippincott; 1972.

14. Dick MJ. Assessment and measurement of acute pain. J Obstet Gynecol Neonatal Nur 1995;24(9):843-8.

15. Turk DC, Melzack R. Hanbook of pain assessment. New York: The Guilford Press; 1992.

16. Gracely RH. Pain psychophysics. In: Manuk S, (editor). Advances in behavioral medicine. New York: JAI Press; 1985. p. 191-231.

17. Gracely RH, Naliboff BD. Measurement of pain sensation. In: Kruger L, (editor). Pain and touch. San Diego, CA: Academic Press; 1996. p. 243-313.

18. Okada M, Teixeira MJ, Tengan SK, et al. Dor em pediatria. Rev Med (São Paulo) 2001;80(2):135-56.

19. Associação Internacional para o estudo da dor (IASP). 1986.

20. Merskey H, Spear FG. The concept of pain. J Psychosomc Res 1967;11(1):59-67.

21. Leventhal H, Everhart D. Emotion, pain, and physical illness. In: Izard CE, (editor). Emotions in personality and psychopathology. New York: Plenum Press; 1979. p. 263-79.

22. Chapman CR, Feather BW. Effects of diazepam on human pain tolerance and pain sensitivity. Psychosom Med 1973;35(4):330-40.

23. Clark WC, Yang JC. Acupunctural analgesia? Evaluation by signal detection theory. Science 1974;184(4141):1096-8.

24. Yang JC, Clark WC, Ngai SH, et al. Analgesic action and pharmacokinetics of morphine and diazepam in man: an evaluation by sensory detection theory. Anesthesiology 1979;51(6):495-502.

25 . Chapman CR. Sensory decision theory methods in pain research: a reply to Rollman. Pain 1977;3(4):295-305.

26. Rollman GB. Signal detection theory measurement of pain: a review and critique. Pain 1977;3(3):187-211.

27. Coppola R, Gracely RH. Where is the noise in SDT pain assessment? Pain 1983;17(3):257-66.

28. Gracely RH, Dubner R. Pain assessment in humans -- a reply to Hall. Pain 1981;11(1):109-20.

29. Gracely RH, Dubner R. Reliability and validity of verbal descriptor scales of painfulness. Pain 1987;29(2):175-85.

30. Hall W. On "ratio scales of sensory and affective pain descriptors". Pain 1981;11(1): 101-7.

31. Rainville P, Carrier B, Hofbauer RK, et al. Dissociation of sensory and affective dimensions of pain using hypnotic modulation. Pain 1999;82(2):159-71. 
32. Rainville P, Duncan GH, Price DD, et al. Pain affect encoded in human anterior cingulate but not somatosensory cortex. Science 1997;277(5328):968-71.

33. Bushnell MC. Psychophysical and brain imaging approaches to the study of clinical pain syndromes. Can J Anaesth 2002;49:R1-R5.

34. Hofbauer RK, Rainville P, Duncan GH, et al. Cortical representation of the sensory dimension of pain. $\mathrm{J}$ Neurophysiol 2001;86(1):402-11.

35. Schnitzler A, Ploner M. Neurophysiology and functional neuroanatomy of pain perception. J Clin Neurophysiol 2000;17(6):592-603.

36. Villemure C, Bushnell MC. Cognitive modulation of pain: how do attention and emotion influence pain processing? Pain 2002;95(3):195-9.

37. Petrovic $P$, Ingvar $M$. Imaging cognitive modulation of pain processing. Pain 2002;95(1-2):1-5.

38. Hoffman HG, Sharar SR, Coda B, et al. Manipulating presence influences the magnitude of virtual reality analgesia. Pain 2004;111(1-2):162-8.

39. Keogh E, Arendt-Nielsen L. Sex differences in pain.
Eur J Pain 2004;8(5):395-6.

40. Craft RM, Mogil JS, Aloisi AM. Sex differences in pain and analgesia: the role of gonadal hormones. Eur $\mathrm{J}$ Pain 2004;8(5):397-411.

41. Patrick L, D'Eon J. Social support and functional status in chronic pain patients. Can J Rehabil 1996;9(2):195-201. 42. Strong J, Ashton R, Chant D. Pain intensity measurement in chronic low back pain. Clin J Pain 1991;7(3):209-18.

43. Turk DC, Okifuji A. Assessment of patients' reporting of pain: an integrated perspective. Lancet 1999;353(9166):1784-8.

44. Anand KJS, McGrath PJ. Pain in Neonates. The Netherlands: Elsevier; 1999.

45. Jamison RN, Rudy TE, Penzien DB, et al. Cognitive-behavioral classification of chronic pain: replication and extension of empirically derived patient profiles. Pain 1994;57(3): 277-92.

Apresentado em 27 de julho de 2010.

Aceito para publicação em 01 de março de 2011. 